\title{
TOPICS IN BLACK HOLE PRODUCTION
}

\author{
Vyacheslav S. Rychkov* \\ Institute for Theoretical Physics, University of Amsterdam, \\ 1018XE Amsterdam, The Netherlands
}

\begin{abstract}
We revisit Voloshin's model of multiple black hole production in transPlanckian elementary particle collisions in $D=4$. Our revised computation shows that the cross section to produce $n$ additional black holes is suppressed by $s^{-1}$, rather than being enhanced as was originally found. We also review the semiclassical gravity picture of black hole production from hep-th/0409131 making additional comments about the meaning of wavepacket subdivision.
\end{abstract}

\section{INTRODUCTION}

Black hole $(\mathrm{BH})$ production in trans-Planckian elementary particle collisions $\left(E \gg E_{\text {Planck }}\right)$ has long been considered a theoretical possibility. If TeV-scale gravity scenarios based on large extra dimensions or warped compactifications are realized in nature, this possibility may be realized in practice at future accelerators (see [1 for a recent review). The key question is the cross section of this process, which is usually assumed to be set by the horizon radius of the produced $\mathrm{BH}$ (the so called "geometric cross section" $\sigma \sim \pi r_{h}^{2}$ ).

In this note I would like to discuss two aspects of the $\mathrm{BH}$ production problem relevant for justifying the geometric cross section estimate. First I will explain how to derive this estimate from a controlled semiclassical gravity approximation to the $\mathrm{BH}$ production process, adding some comments to the original discussion of [2] (also recently reviewed in [3]). Then I will estimate the cross section of multiple BH production due to collisions between virtual gravitons emitted by the primary particles, in a model first proposed by Voloshin [4. The conclusion (contrary

*To appear in the proceedings of the Cargèse Summer School "String Theory: From Gauge Interactions to Cosmology", June 7-19, 2004. 
to [4]) is that multiple $\mathrm{BH}$ production gives a subdominant contribution to the total cross section.

The size of produced BHs in large extra dimension scenarios is typically much smaller than the size of extra dimensions, and their production may thus be considered as happening in flat $D$ dimensional spacetime. Since we are focussing on theoretical issues, to keep the discussion clear we will work in $D=4$. We will also use Planck units, setting $E_{\text {Planck }}=1$.

\section{WAVEPACKETS AND SEMICLASSICS}

In this note we will adhere to the standard believe that in quantum theory of gravity classical BHs with mass $M \gg 1$ will be realized as longlived resonance states, decaying via Hawking radiation, with lifetime $\sim M^{3}$. It is then energetically allowed to produce such BHs in transPlanckian $(E \gg 1)$ elementary particle collisions.

To estimate the cross section of this process, Eardley and Giddings [5] looked at the grazing collision of two ultrarelativistic point particles in classical general relativity, using formation of a closed trapped surface (CTS) as a sufficient condition for BH formation. In this totally classical description, lower bound for the cross section is given by $\pi b_{\max }^{2}$, where $b_{\max }$ is the maximal impact parameter for which we are able to find a CTS in the spacetime formed by two colliding Aichelburg-Sexl shock waves. [5] found $b_{\max } \sim E \sim r_{h}$, and this implies $\sigma \sim \pi r_{h}^{2}$. Recourse to such an indirect method is necessary, because explicit solutions of Einstein's equations exhibiting the final BH state are out of reach.

How do we justify this approach from quantum gravity point of view? A point of immediate concern is that particles in a collider experiment are described by wide wavepacket states of macroscopic size (set essentially by the beam radius, which in turn is determined by the focussing ability of accelerator magnets). These wavepackets are vastly larger than $\mathrm{BHs}$ whose production we are trying to describe. If we use the energy momentum tensor of these wavepacket states $|\psi\rangle$ in the RHS of the semiclassical Einstein's equations

$$
R_{\mu \nu}-\frac{1}{2} g_{\mu \nu} R=\left\langle\psi\left|T_{\mu \nu}\right| \psi\right\rangle,
$$

we won't see any BH production whatsoever, since the energy is spread out over a huge volume, and the energy density is insufficient to cause collapse. Informally speaking, particles "do not fit" inside a BH.

However, this does not mean that BHs do not form. The correct interpretation is that the part of the gravitational field wavefunction corresponding to $\mathrm{BH}$ production was erased - averaged away - by eq. (1.1). Some averaging is always inevitable when using the semiclassical field 


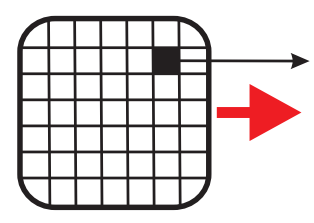

Figure 1.1 Subdivision of the right-moving particle wavepacket. Similar subdivision has to be done for the left-moving particle.

equations, since we substitute $T_{\mu \nu}$ by its expectation value. Unfortunately, in this case it destroys precisely the part of the wavefunction we are interested in.

To see BH production, one should instead proceed as follows. First of all, we have to subdivide the initial wavepackets into much smaller wavepackets of size $w \ll r_{h}$ :

$$
|\psi\rangle=N^{-1 / 2} \sum_{i=1}^{N}\left|\psi_{i}\right\rangle
$$

This subdivision is carried out so that the small wavepackets $\left|\psi_{i}\right\rangle$ in the RHS are almost orthogonal. This orthogonality is quite obvious in the position representation (see Fig. 1.1). Because of the orthogonality, collisions between different pairs of small wavepackets are mutually excluded possibilities, and probabilities of $\mathrm{BH}$ production in each such elementary collision should be added. Now, it is the collisions of the small wavepackets that we are going to analyze using eq. (1.1). Condition $w \ll r_{h}$ ensures that the small wavepackets produce a collision spacetime which is a small perturbation of the one corresponding to point particles of the same energy. Thus the Eardley-Giddings analysis applies, and adding probabilities results in the geometric cross section.

Using finite-size wavepackets instead of point particles has an additional bonus in that it puts the conditions of applicability of the semiclassical approximation under control. For example, curvature blows up when the shock fronts of Aichelburg-Sexl waves corresponding to point particles collide 6, 7]. However, taking wavepacket size into account regulates the curvature and brings it below the Planck value, so that we can trust the semiclassical gravity approximation [2].

\section{MULTIPLE BLACK HOLE PRODUCTION}

According to the above discussion, the geometric cross section formula provides a lower bound for a single large $\mathrm{BH}$ production cross section in a trans-Planckian collision. However, as the energy of the particles grows, multiple $\mathrm{BH}$ production also becomes energetically allowed. It is 


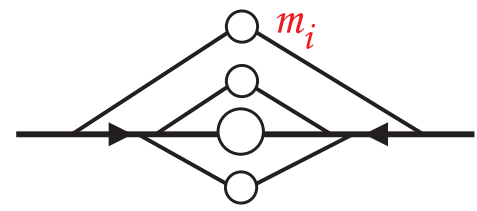

Figure 1.2 A typical diagram for multiple BH production in Voloshin's model

important to understand which process is dominant at asymptotically high energies. If multiple $\mathrm{BHs}$ dominate, it will be much harder to observe dipole patterns of emitted particles expected in the Hawking evaporation of a single large $\mathrm{BH}$.

We are going to discuss a model proposed by Voloshin 4, in which multiple BHs are produced due to collisions between virtual gravitons emitted by the trans-Planckian projectiles. Such virtual graviton emission is a quantum effect: classically, any radiation happens after the particles collide. The process is studied diagrammatically, with a typical diagram shown in Fig. 1.2. Only the case of small peripheral BHs $\left(1 \ll m_{i} \ll E, i=1 \ldots n\right)$ is considered, so that the gravitons are "soft".

The production amplitude is computed from the diagrams using the standard QFT propagators and particular vertices for soft graviton emission and for BH production (Fig. 1.3). The amplitude to emit a positive helicity graviton of energy $\omega \ll E$ and small transverse momentum $\mathbf{k}=\left(k_{2}, k_{3}\right),|\mathbf{k}| \ll \omega$ is given by (see Appendix A)

$$
A \propto(E / \omega)^{2}\left(k_{2}+i k_{3}\right)^{2} .
$$

(We will not pay attention to constant numerical factors. Thus our final result (1.5) is valid up to a factor $c^{n}$.)

Although we are unable to compute the elementary $\mathrm{BH}$ production vertex $f\left(q^{2}\right)$, the geometric cross section allows to fix the combination

$$
\left|f\left(q^{2}\right)\right|^{2} \rho\left(q^{2}\right) \sim\left(q^{2}\right)^{2},
$$

where $\rho\left(q^{2}\right)$ is the density of BH states at mass $\sqrt{q^{2}}$.

A crucial final element of the model is a condition which ensures that the emitted gravitons do not subsequently fall into a common large $\mathrm{BH}$.

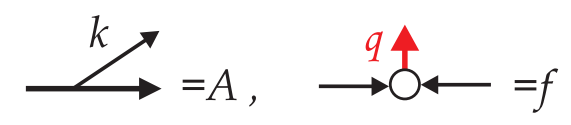

Figure 1.3 Graviton emission and $\mathrm{BH}$ production vertices 
Such an infall may happen due to graviton rescattering diagrams, which we are not going to compute. Thus, without a "fall safe" condition we would be in danger of greatly overestimating the multiple BH amplitude. Voloshin's "fall safe" condition limits the transverse momenta of emitted gravitons by $|\mathbf{k}| \lesssim 1 / E$.

To derive this condition, we note that a typical emitted graviton will be off-shell by $\Delta E \sim \mathbf{k}^{2} / \omega$. It will exist for a time interval $\Delta t \sim 1 / \Delta E$, during which it will reach transverse separation $\Delta z \sim(\mathbf{k} / \omega) \Delta t$ from the projectile. Voloshin's condition arises if we require that this transverse separation is larger than the horizon radius of the big $\mathrm{BH}$ formed in the collision of the primary particles: $\Delta z \gtrsim E$.

In the described model, our computation (see Appendix B) gives the following amplitude to produce $n$ additional BHs with 4-momenta $q_{i}$ :

$$
f^{(n)}\left(s, q_{i}^{2}\right) \sim f(s) \prod_{i=1}^{n}\left(q_{i}^{2}\right)^{-2} f\left(q_{i}^{2}\right)
$$

The original computation of [4, Eq. (5), gave an amplitude larger than (1.5) by a factor of $\left(s q_{i}^{2}\right)^{n}$. We believe that our result is correct; see Appendix B for an explanation.

Using (1.4), we can compute from (1.5) the contribution of the diagrams from Fig. 1.2 into the total cross section $\sigma_{n}$ to produce one large $\left(m^{2} \sim s\right)$ and $n$ small BHs. This contribution will behave like $s^{1-n}$, the suppression being due to the phase space restriction $|\mathbf{k}| \lesssim 1 / E$ satisfied by the small $\mathrm{BHs}$ as a consequence of the "fall safe" condition.

However, for $n \geq 2$ there are diagram which give a larger contribution, so that $\sigma_{n} \sim$ const is likely for any $n \geq 1$. Consider, e.g., Fig. 1.4 where the primary particles emit "fall safe" gravitons of energy $E_{1}, 1 \ll$ $E_{1} \ll E$, and it is these gravitons that form $n$ smaller BHs according to the previous model. The allowed phase space for this diagram will be much bigger, since the individual small BHs can now have much larger transverse momenta $|\mathbf{k}| \lesssim 1 / E_{1}$, only their sum being $\lesssim 1 / E$. Choosing $E_{1}$ above the threshold of $n \mathrm{BH}$ production, we will get an $s$-independent contribution to $\sigma_{n} \cdot{ }^{1}$

In any case, we see that $\sigma_{n}$ is suppressed compared to the geometric cross section value $\sigma_{0} \sim s$.

${ }^{1}$ It is also easy to see that the inclusive cross section of multiple $\mathrm{BH}$ production cannot decay with $s$. This is because the particles may first reduce their energy by emitting one or more "fall safe" gravitons (which costs no $s$-dependent factor, see [4, Eq. (3)), and then collide to form BHs. I am grateful to M. Voloshin for this remark. 


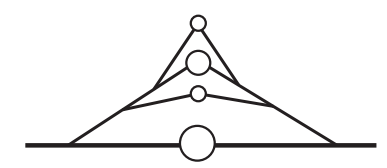

Figure 1.4 A diagram giving an $s$-independent contribution to $\sigma_{n}$

\section{CONCLUSIONS}

In this note we discussed two aspects of the $\mathrm{BH}$ production problem. In Section 2 we discussed how wavepacket arguments can be used to justify the use of semiclassical gravity in this problem. In Section 3, we revisited Voloshin's model of multiple $\mathrm{BH}$ production, and presented a revised computation which shows that this process is suppressed compared to the production of a single large BH. Our main conclusion is that the geometric cross section estimate seems to be in rather good health, surviving all checks and resisting any disproving attempts.

\section{Acknowledgments}

I would like to thank Steve Giddings for the opportunity to collaborate on 22. A part of Section 2 arose as an answer to Marco Cavaglia's interesting questions. I am especially grateful to Misha Voloshin for the e-mail correspondence which helped me to understand his model, and for the critical remarks about the early versions of the computation. It is also a pleasure to thank the organizers of the Cargèse 2004 String Theory school. This work was supported by Stichting FOM.

\section{Appendix: A. Soft graviton emission vertex}

Consider emission of a positive helicity collinear graviton with energy $\omega$ and small transverse momentum $\mathbf{k}=\left(k_{2}, 0\right), k_{2} \ll \omega$. Its polarization tensor, satisfying the constraints $h_{i j} k_{j}=0, h_{i i}=0$, is given by

$$
h_{i j} \approx\left(\begin{array}{ccc}
k_{2}^{2} / \omega^{2} & -k_{2} / \omega & -i k_{2} / \omega \\
-k_{2} / \omega & 1 & i \\
-i k_{2} / \omega & i & -1-k_{2}^{2} / \omega^{2}
\end{array}\right) .
$$

The emission amplitude is $A \propto\left\langle p-k\left|T_{i j}\right| p\right\rangle h_{i j}$, where the energy momentum tensor matrix element is

$$
\left\langle P\left|T_{\mu \nu}\right| p\right\rangle=\left\langle P\left|\phi_{, \mu} \phi_{, \nu}-\frac{1}{2} \eta_{\mu \nu}(\partial \phi)^{2}\right| p\right\rangle=p_{\mu} P_{\nu}+p_{\nu} P_{\mu}-\eta_{\mu \nu}(p P) .
$$

From this we find $A \propto E^{2} k_{2}^{2} / \omega^{2}$. 


\section{Appendix: B. Multiple BH production amplitude}

We will compute the $n=2$ amplitude in the case when both small BHs are produced at rest in the c.m. frame: $q_{i}=\left(m_{i}, 0,0,0\right)$ in Fig. 1.B.1 The calculation of the amplitude naturally splits into 3 steps. First,

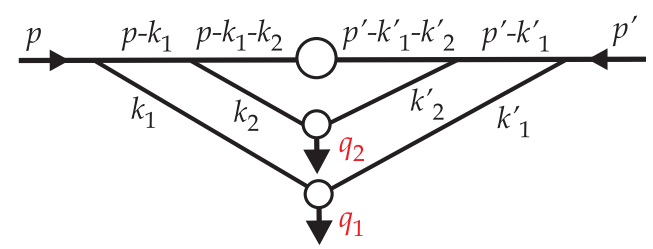

Figure 1.B.1 A diagram for one large and two small $\mathrm{BHs}$ in the final state

we compute the loop integral over the longitudinal momenta. Then, we multiply by the emission vertices and integrate over the transverse momenta. Finally, we multiply by the $\mathrm{BH}$ production vertices.

It is convenient to write the graviton momenta as follows $(i=1,2)$ :

$$
k_{i}=\left(\frac{m_{i}}{2}+\frac{x_{i}-y_{i}}{2}, \frac{m_{i}}{2}-\frac{x_{i}+y_{i}}{2}, \mathbf{k}_{i}\right), \quad k_{i}^{\prime}=q_{i}-k_{i} .
$$

The longitudinal loop integral now separates into the integrals over $x_{i}$ and $y_{i}$. The part depending on $x_{i}$ is

$$
\begin{aligned}
I & =\int d x_{1} d x_{2} \frac{1}{k_{1}^{2} k_{2}^{2}\left(p-k_{1}\right)^{2}\left(p-k_{1}-k_{2}\right)^{2}} \\
& \approx \int \frac{d x_{1} d x_{2}}{\left(m_{1} x_{1}-\mathbf{k}_{1}^{2}\right)\left(m_{2} x_{2}-\mathbf{k}_{2}^{2}\right)\left(-E x_{1}-\mathbf{k}_{1}^{2}\right)\left(-E\left(x_{1}+x_{2}\right)-\mathbf{k}_{12}^{2}\right)} .
\end{aligned}
$$

The $+i \epsilon$ is implicit in each denominator. This integral is easy to compute by closing the contour in the lower half-plane. Omitting corrections of the order $m / E \ll 1$, we have

$$
I \propto\left[E^{2} \mathbf{k}_{1}^{2}\left(\mathbf{k}_{1}^{2}+\mathbf{k}_{2}^{2}\right)\right]^{-1}
$$

Since we used complex analysis, it is important to check that the integral is dominated by soft, almost real gravitons. This is indeed true, the important region being $\left|x_{i}\right| \lesssim \mathbf{k}_{i}^{2} / m_{i}$. The tail corresponding to $\left|x_{i}\right| \gg \mathbf{k}_{i}^{2} / m_{i}$ can be estimated directly as:

$$
\frac{1}{m_{1} m_{2} E^{2}} \int \frac{d x_{1}}{x_{1}^{2}} \frac{d x_{2}}{x_{2}^{2}} \ll I
$$


This check also justifies post factum neglecting the dependence of the graviton emission vertices on $x_{i}$, as well as the omission of $x^{2}$ terms in the denominators of (1.B.2).

Before proceeding to the next step, we have to add diagrams differing by the order of graviton emission (Fig. 1.B.2). This summation has the

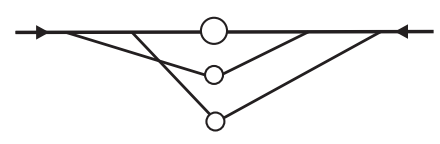

Figure 1.B.2 One of the 3 permuted diagrams

effect $I \rightarrow\left[E^{2} \mathbf{k}_{1}^{2} \mathbf{k}_{2}^{2}\right]^{-1}$. Finally, we multiply by the same factor arising from the $y$-integration, and get:

$$
\left[E^{2} \mathbf{k}_{1}^{2} \mathbf{k}_{2}^{2}\right]^{-2} \quad \text { (longitudinal part) . }
$$

We will assume that the small BHs are produced in the spin 0 state, so that the colliding gravitons have opposite helicities. The product of the corresponding emission vertices is

$$
\propto(E / m)^{4}\left(k_{2}+i k_{3}\right)^{2}\left(k_{2}-i k_{3}\right)^{2}=(E / m)^{4}\left(\mathbf{k}^{2}\right)^{2} .
$$

Multiplying (1.B.5) by such factors for both BHs and integrating over $\left|\mathbf{k}_{i}\right| \lesssim 1 / E$ (the "fall safe" condition), we get a number $\sim\left(m_{1} m_{2}\right)^{-4}$.

Finally, we multiply by the $\mathrm{BH}$ production vertices and arrive at

$$
f^{(2)} \sim f(s) f\left(m_{1}^{2}\right) f\left(m_{2}^{2}\right)\left(m_{1} m_{2}\right)^{-4} .
$$

This formula agrees with the general result (1.5) in the considered case.

Extension of the above computation to the general case is quite straightforward. A point worth mentioning is the use of the standard identity

$$
\sum_{p e r m}\left[a_{1}\left(a_{1}+a_{2}\right) \cdots\left(a_{1}+\ldots+a_{n}\right)\right]^{-1}=\left[a_{1} \cdots a_{n}\right]^{-1}
$$

when summing over the order of graviton emission in the $n>2$ case.

In [4], the estimate $\int d^{4} k_{i} /\left(k_{i}^{2} k_{i}^{\prime 2}\right) \sim O(1)$ was used in computing the amplitude. However, our analysis shows that the correct estimate is:

$$
\int \frac{d^{4} k}{k^{2} k^{\prime 2}} \propto \int \frac{d x d y d^{2} \mathbf{k}}{\left(m x-\mathbf{k}^{2}+i \epsilon\right)\left(m y-\mathbf{k}^{2}+i \epsilon\right)} \sim \frac{1}{m^{2} E^{2}} .
$$

This extra factor, for each of $n$ BHs, explains the difference between (1.5) and the result of [4. 


\section{References}

[1] P. Kanti, "Black holes in theories with large extra dimensions: A review," arXiv:hep-ph/0402168.

[2] S. B. Giddings and V. S. Rychkov, "Black holes from colliding wavepackes," arXiv:hep-th/0409131.

[3] V. S. Rychkov, "Classical black hole production from quantum particle collisions", to appear in the proceedings of the 6th Alexander Friedmann International Seminar on Gravitation and Cosmology, Cargése, June 28-July 3, 2004, arXiv:hep-th/0410041.

[4] M. B. Voloshin, "More remarks on suppression of large black hole production in particle collisions," Phys. Lett. B 524, 376 (2002), arXiv:hep-ph/0111099.

[5] D. M. Eardley and S. B. Giddings, "Classical black hole production in high-energy collisions," Phys. Rev. D 66, 044011 (2002), arXiv:gr-qc/0201034.

[6] V. S. Rychkov, "Black hole production in particle collisions and higher curvature gravity," Phys. Rev. D 70, 044003 (2004), arXiv:hep-ph/0401116.

[7] V. S. Rychkov, "Tests of classical gravity description for microscopic black hole production," arXiv:hep-ph/0405104. 